\title{
Immersive Digital Games: The Interfaces for Next-Generation E-Learning?
}

\author{
Michael D. Kickmeier-Rust ${ }^{1}$, Neil Peirce ${ }^{2}$, Owen Conlan ${ }^{2}$, \\ Daniel Schwarz ${ }^{3}$, Dominique Verpoorten ${ }^{4}$, and Dietrich Albert ${ }^{1}$

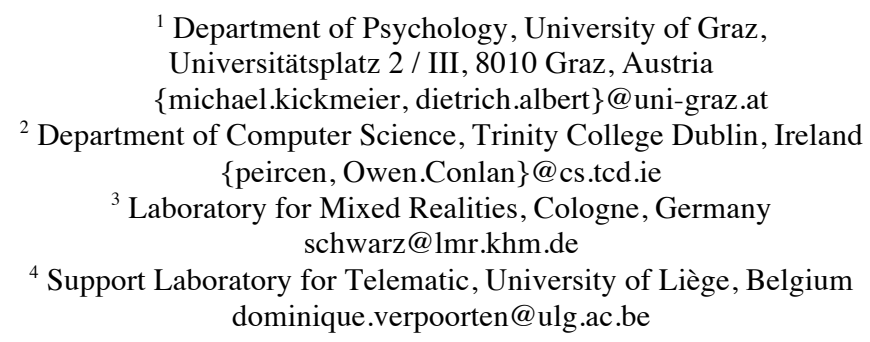

\begin{abstract}
The intrinsic motivation to play, and therefore to learn, that might be provided by digital educational games teases researchers and developers. However, existing educational games often fail in their attempt to compete with commercial games and to provide successful learning. Often some learning is added to digital games or some gameplay is added to educational applications. Successful education games, however, require merging professional game design with sound pedagogical strategies. This merge creates a new and hybrid format that truly deserves the denotation being a learning game. Moreover, a methodology is required that allows continuously balancing gaming and learning challenges and the learner's abilities and knowledge in order to retain an immersive gaming experience. In this article we introduce approaches to game design and didactic design, as well as a framework for adaptive interventions in educational games.
\end{abstract}

Keywords: Digital educational games, Game Design, Didactic Design Adaptive intervention, Microadaptivity, Competence-based Knowledge Space Theory

\section{Introduction}

Over the past decades e-learning and technology enhanced learning and teaching, has become more and more popular. Consequently, a vast number of e-learning platforms and multimedia learning objects are available and widely accepted for the use in schools as well as for continuing education. E-learning has high potential to distribute high quality learning objects, facilitating learning and enabling new perspectives and insights to learning content.

However, the majority of e-learning and multimedia learning objects are based on traditional 2D user interfaces, e.g. website interfaces, Flash animations, Java applets, 
or PDF files. Provocatively speaking, current e-learning offers have all more or less the same unexciting look and feel. This perspective is compounded by the proliferation of immersive computer games that learners are exposed to outside of learning experiences. Moreover, independent of usability and accessibility, traditional interfaces for educational applications have serious weaknesses from the perspectives of learning psychology and didactics. For example, they are not intrinsically motivational and it is difficult to retain a learner's interest, it is difficult to provide a meaningful context throughout learning episodes, it is difficult to activate prior knowledge as a basis for learning, it is not always possible to provide real-world problems for practicing new knowledge, and meaningful application of new knowledge is difficult without a meaningful and engaging context [1].

Immersive educational computer games offer a highly promising approach to overcome the mentioned weaknesses and to make learning more engaging, satisfying, and probably more effective. Currently, there is significant hype over game-based learning, ranging from edutainment to games for primarily educational purposes (see [2] for an overview). The major strength of digital games in education is a high level of intrinsic motivation to play and to proceed in the game and, thus, to learn within the context of a meaningful and continuous storyline and the according para-scoial dimension for both gaming and learning provided by game characters. According to Malone [3], the factors forming that strength and making games fun are challenge, fantasy, and curiosity. Educational games provide clear goals and rules, a meaningful learning context, an engaging storyline, immediate feedback, a high level of interactivity, challenge and competition, random elements of surprise, and rich and appealing learning environments [3], [4]. These factors determine motivation to play and to learn but are also considered to be important for successful and effective learning, e.g. interactivity, feedback, problem solving, or context effects (for reviews see [1], [5]). On the other hand, educational games have also major disadvantages like difficulties in providing appropriate balance between gaming and learning activities, providing a continuous balance between challenge and ability, aligning the game with national curricula, or the extensive costs of developing high quality games (for a review see [6]). Due to these problems, most of today's educational games cannot compete with their commercial counterparts in terms of gaming experience, immersive and responsive environments and storytelling, or intrinsic motivation to play. Moreover, most educational games do not rely on sound instructional models thus leading to a separation of learning from gaming and often they provide gaming actions only as reward for learning. Therefore, such games do not differ significantly from other traditional multimedia learning applications.

The ELEKTRA project (www.elektra-project.org) has the ambitious and visionary goal to fully utilize the advantages of computer games and their design fundamentals for educational purposes and to address and eliminate the disadvantages of gamebased learning as far as possible. Nine interdisciplinary European partners contribute to the development of a sound methodology for designing educational games and the development of a comprehensive game demonstrator based on a state-of-the-art 3D adventure game teaching physics according to national curricula. Furthermore, ELEKTRA will address important research questions concerning game design, didactic design, or adaptive interventions. 


\section{Game Design}

In the point of view of many game designers, educationists, and psychologists, the genre of digital games offers a wide range of means of expressions to enhance the presentation of educational contents and to provide learners with an exciting and new interface to knowledge.

In the disciplines of game design and development exists a long tradition and experience in the creation of immersive narratives and story lines as well as in the design of appealing virtual environments. Design domains include game play design, story design, visual design, information design, sound design, interface design, and interaction design, and each is a fine art. When seriously aiming for integrating educational purposes to digital games and developing primarily educational games whose main goal is to convey educational content, the mentioned design domains are facing completely new challenges and demands. On the other hand didactic designers for educational software are confronted with the design potential of a highly interactive spatiotemporal game world that goes far beyond the interfaces of traditional e-learning applications. Therefore the establishment of a new genre is required within which both worlds, recreational games and psycho-pedagogical aims and methods, are meld into a new hybrid format. From an educational perspective, digital games offer (a) a real-time 3D virtual environment that serves as space for learning and is (b) perceived by the learner through a user-centric perspective ("Egoperspective"). Such environment is determined by (c) situations. In the format of digital educational games basically three types of situations exist: (a) learning situations (LeS) with the particular aim of teaching learning matter, (b) gameplay situations that provide interactions with the game environment, objects, and characters requiring a skilful usage of tools and artfully accomplished game moves, and (c) story situations that are required to combine learning and gameplay situations in a meaningful context that propels the learning game experience with the motivation of a enthralling story and its characters. The different situations and types of situations must be joined, motivated, and legitimated by a global narrative that provides a meaningful context.

Still, a large number of open questions remain to be addressed by research in the ELEKTRA project and beyond the project. Just to mention a few, these questions concern an appropriate balance of gameplay and learning, the translation of game elements into a pedagogically sound learning methods, or the role of para-social interaction play (either with real mates or with non-player characters; NPC).

\section{Didactic Design}

Besides an immersive and motivating game design, a sound pedagogical approach is required to develop a successful educational game. Up-front choice of reference pedagogical frameworks allowing instructionally informed decisions is a key success factor when developing any kind of virtual learning environments [7]. This emphasis is taken up by authors in the field of digital game-based learning [4], [8], [9]. Without such educational beacons, risks are high of lacking vocabulary for describing and 
implementing pedagogical endeavors, ultimately loosing instructional focus. This section briefly presents the dominant conceptual tool used in ELEKTRA for dealing with methods of learning. The model is tentatively connected to Bloom's taxonomy of educational objectives [10]. The articulation of methods, objectives, and evaluation is conceptually scrutinized according to the principle of "triple consistency" which is seen, in ELEKTRA's interdisciplinary perspective, as an apparently basic but major concern and contribution of traditional pedagogy to the whole project.

Created in a context of teacher professional development ([11], [12], the 8 Learning Events Model (8LEM) is intended to trigger reflections of practitioners about methods of learning and their diversification. This emphasis on methods is grounded in an educational philosophy which postulates that any educational action cannot restrict itself to "products of learning" but must also look at "processes of learning". Conceived for inviting teachers to broaden their repertoire of methods, 8LEM helps managing in a more systematic way the tricky theoretical and practical issue of designing equivalent but different (inter-individual diversification) or complementary (intra-individual diversification [13]) learning/gaming paths and events, regardless whether they are instantiated along an adaptation or an adaptivity mode. The learning events specified in 8LEM are exploration, imitation, reception, creation, experimentation, practice, debate, and meta-reflection.

Methods, adapted or not, do not take place in a vacuum. They become meaningful once they are connected to learning objectives [14]. In this respect, and still in order to making the instructional design and its rationale [15] to the designer and possibly to users, each 8LEM-labelled activity is attached to the level of Bloom's taxonomy [10] it is deemed to serve. The taxonomy includes learning objectives like knowledge, understanding, application, analysis, synthesis, and evaluation. The resulting combinations of learning events and objectives result in 48 learning activities. This gives a rough but complete overview of what is pedagogically at stake in the virtual learning environment. Diversification of methods can be identified as well as levels of cognitive objectives targeted.

An additional important component of ELEKTRA's pedagogical conceptual toolkit supporting game development is the notion of "triple consistency". This cornerstone principle of valid pedagogy stresses the mandatory solidarity which must exist between objectives, methods and evaluation. Tyler [16] demonstrated that failures in learning system are related to a lack of consistency in between learning objective, method used to teach, and evaluation of the level of objective's knowledge reached.

\section{Adaptive Interventions}

An immersive digital game for educational purposes requires a subtle balance of challenge and ability. Thus, the game must be able to adapt to the learner's knowledge, skills, and abilities, motivation, and also pedagogical implications. Generally, adaptive approaches to e-learning contest the one-size-fits-all approach of traditional learning environments, trying to tailor the learning environment according to individual needs and preferences. Adaptivity refers to navigation, curriculum 
sequencing, and presentation. For example, an adaptive system may only provide learning objects which are suitable for an individual's learning progress - too difficult and also too easy learning objects might not be displayed in order to avoid visual and cognitive load and to suggest an appropriate learning path through the learning content. In the context of immersive digital games, existing approaches to adaptivity must be extended in order to retain an immersive gaming experience, motivation, and probably flow experience by suitable adaptive interventions.

\subsection{Adaptive Influencing Learning Situations in a Game (and not breaking the narrative)}

Narratives and storylines have long been known to be a major motivating factor in video games. Contemporary games frequently employ a game narrative as a motivating factor. Examples can found through out all video game genres and include many popular game franchises such as Tomb Raider, Command \& Conquer, Grand Theft Auto, Super Mario, and Final Fantasyl to name but a few. Evidently the presence of a plausible narrative within a video game can lead to an engrossing and rewarding experience. It is for this reason that the ELEKTRA demonstrator has an embedded narrative that has been crafted to appeal to the target audience of 13-15 year of school pupils. Whereas an embedded narrative has major advantages for motivating a learner to engage, it also poses considerable challenges for educational adaptation within the game. Educationally adaptive systems to date have focused largely on adaptively ordering and presenting learning tasks. Within an educationally adaptive game such as ELEKTRA the learning tasks are so integrated with the games narrative that the reordering of learning tasks would result is a corresponding reordering of narrative plot elements. With a linear narrative this would result in a nonsensical narrative that is implausible. The challenge of creating dynamic yet plausible adaptive narratives is considerable and requires arduous manual editing of branching narratives. Experimental systems such as Façade [17] exemplify the difficulties of creating adaptive narratives.

Within the field of adaptive hypermedia adaptation is limited by the presentation medium and so adaptation is manifested through intermittent curriculum ordering and adaptive presentation (e.g., [18]). Due to the nature of 3D immersive games the adaptation within the ELEKTRA demonstrator needs to be continuous and less periodic; it needs to occur at a greater frequency than on a task by task level. Considering this with the existing difficulties associated with generating adaptive narratives, the ELEKTRA game provides microadaptivity, that is, adaptation within learning tasks as opposed to around them.

Microadaptivity removes the challenges of adaptive narratives yet creates challenges of its own due to the nature of the experience of game play, and the impact that game world changes can have on a player's experience. Games are considered to be intrinsically rewarding to play, and it is factors such as self-governance,

\footnotetext{
${ }^{1}$ Tomb Raider, Core Deign (1996); Command and Conquer, Westwood Studios (1995); Grand Theft Auto, DMA Design (Rockstar North) (1997); Super Mario Bros., Nintendo (1983); Final Fantasy, Square Enix (1987)
} 
immersion, clear goals, immediate feedback, and game feasibility that contribute to rewarding play. Any adaptation within the game world must thus be achieved in such a manner so that it is not to the detriment of the play experience. Essentially the experience of Flow [19] must be maintained, where the learner is immersed, selfgoverning, aware of their ability to succeed, and clearly able to see the game's goal. From an educational perspective the experience of Flow is rarely catered for in adaptive eLearning systems. There is evidence however to suggest that Flow can be beneficial to learning in classroom environments [20] and may prove beneficial to learning experiences in immersive educational games.

\subsection{Non Invasive Microadaptivity}

Achieving microadaptivity without compromising the learner's gaming experience requires that any adaptation must be achieved in consideration of the gaming experience. To accomplish this within ELEKTRA the Learning Engine, the system responsible for adaptation, does not directly intervene with the game engine but provides recommendations for changes that are desirable. These recommendations are always contextually specific to a learning situation and are manifested in such a way that they are in line with the game's narrative and the learner's sense of selfgovernance. The intention of the Learning Engine as to what needs to be adapted is embodied by the recommendations. However, it is at the ultimate discretion of the game engine as to whether or not to enact a recommendation, this is provided as an extra insurance so that no adaptation is detrimental to the learner's gaming experience.

It is through these appropriate recommendations that the Learning Engine can effectively influence the game engine to adapt without negatively impacting the game's narrative. The ultimate realization of the recommendations is determined by the game engine and they can take the form of hinting or guidance from a NPC, motivational support from an NPC, throttling the learning situation difficulty, or appropriate direct intervention by an NPC where deemed necessary.

\subsection{Abstraction within the Learning Engine}

The ELEKTRA game consists of a number of LeS each of which may be adapted to the learner's performance. The generation of adaptive rules for each learning situation independently would be both time consuming and lead to many rules that are similar in nature yet sufficiently LeS specific to prevent their reuse. This is undesirable from an authoring perspective as it is both arduous and difficult to author adaptation for each learning situation; additionally the reuse of adaptive pedagogical strategies is hindered. The ELEKTRA demonstrator overcomes this through a system of abstracted pedagogical adaptation. Whereas with many adaptive educational systems the adaptation for a learning situation is specific to that situation only, the Learning Engine provides generic abstracted pedagogical adaptation that is applied to all learning situations. This is then mapped into recommendations that are specific to a learning situation. It is in this mapping from generic adaptation to specific game 
changes that allows the Learning Engine adaptively influence a learning situation without adversely affecting the game's narrative.

The learning engine's adaptation methodology can be broken into four separate components, broadly described as Evidence, Inferring, Recommendation, and Realization. The first component, Evidence, is that information coming from the game engine that is deemed pertinent to the learner's performance within a learning situation. The second component, Inferring, embodies an assessment of the Evidence information and the generation from this of generic semantically meaningful performance information. The Recommendation component consumes the output of the Inferring component in order to generate a generic pedagogical adaptation goal. The final component the Realization consumes generic pedagogical goals and produces learning situation specific adaptation within the game engine to achieve the generic goal.

The advantages of this specific-generic-specific cycle can be found in the ability of the system to reuse pedagogical rules, reduce the specific per learning situation adaptation authoring, and aid the independent authoring of both pedagogical adaptation and game learning situations.

In ELEKTRA the above cycle uses skills assessment at the Inferring component as the key means for moving from the specifics of a LeS to the generic capability to reason pedagogically about the learner's performance. For example, based on the learner's interactions with a learning situation evidence is produced for how they are progressing. This evidence is translated into their acquisition of skills pertinent to the domain in which the LeS is set. With no knowledge of the specific LeS, recommendations are made based on this inferred progress. These recommendations are pedagogically informed and indicate the type and level of influence required. It is then the responsibility of the Game Engine to determine how this recommendation will be realized.

\subsection{The Cognitive Framework Behind Adaptivity}

The cognitive framework underlying adaptivity in ELEKTRA is CbKST. In its original formulation, Knowledge Space Theory (KST) [21] provides a set-theoretic framework for organizing domain of knowledge and for representing the knowledge of individuals. The basic idea behind KST is to assume prerequisite relations among a finite set of problems (e.g., test-items). If a problem $a$ is a prerequisite for a problem $b$, from mastering problem $b$ also the mastering of problem $a$ can be inferred. Based on the set of problems and the corresponding prerequisite relation a knowledge structure is established which includes all admissible or meaningful knowledge states. Each individual can be assigned to a specific knowledge state.

The behavioral KST was extended by explicitly referencing the latent skills and competencies underlying the observable behavior [22], [23], [24]. On of the most successful approaches to CbKST is the Competence Performance Approach (CPA) by Klaus Korossy [25], [26]. The basic idea of CPA is to assume a basic set $E$ of abstract cognitive skills that are relevant for mastering the problems of a domain. The skill state of an individual is the collection of all available skills, which is not directly observable but can be uncovered on the basis of the observable performance on the 
problems representing the domain. As in KST, prerequisite relations are defined on the set of skills establishing a skill structure $C$, which contains all possible skill states. Utilizing skill and problem functions, families of subsets of skills can be mapped to problems, which can be mastered with the given set of skills and vice versa. By the assignment of skills to the problems of a domain, also a performance structure or problem structure on the set of problems is induced.

An example is given in Fig. 1. If we assume that skill $s 1$ is a prerequisite for skill $s 2$, skill states, which include skill $s 2$ but not all of its prerequisites, in this example $s 1$, are not admissible, at least from a deterministic perspective. This procedure allows us to substantially reduce the number of possible skills states. Without prerequisite relation, six skills establish a skill structure that includes 64 skill states, i.e. the power set of all skills $\left(2^{6}\right)$. The prerequisite relation in Fig. 1 establishes a skill structure that includes only eleven possible skill states. A major advantage is that CPA allows multiple, individual, not necessarily linear learning paths. This offers a sound model of learning objectives which, in turn, establishes the very basis for game design as well as learning design. Moreover, by an assignment of latent skills to LeS and single objects (e.g., tools, switches, laboratory equipment, or books) within LeS using problem and skill functions, the skill structure serves a basis for reasoning about the skill state of a learner on the basis of the performance in LeS.

Adaptively providing a learner with suitable learning objects is a major mechanism of existing approaches to adaptive e-Learning. This principle can also be utilized for providing adaptivity on a macro-level in digital educational games. In the context of game design, branched storylines for interactive, personalized storytelling could be realized, characterized by multiple paths through the game environment. As discussed in section 4.1 , in the context of educational games the principle of macroadaptivity has major drawbacks, however. First, it might be highly expensive to implement the requirements of adaptive storytelling, which is a demand of an adaptive ordering of LeS. Second, a plausible re-ordering might not be possible in a linear storyline and third, a theoretical model for adaptive storytelling still has to be developed. For these reasons, in the ELEKTRA project we pursue an approach to adaptivity on a microlevel.
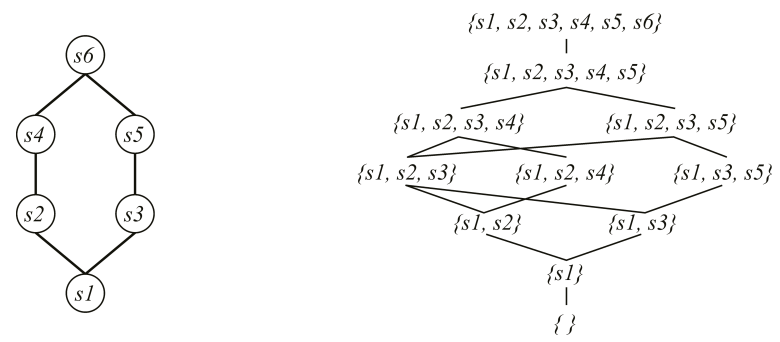

Fig. 1. Prerequisite relation (left) and the corresponding skill structure (right).

In order to meet the requirements of non invasive adaptive interventions, traditional CbKST-based approaches to the assessment of skills and competencies must be complemented with an approach of probabilistic competence diagnosis. In contrast to traditional approaches to adaptive knowledge assessment, which is mostly 
based on typical test items (e.g., a multiple choice task), adapting to a learner's skills on a micro-level requires a probabilistic assessment of knowledge based on the learner's actions within a LeS. Such LeS include a number of objects, which can be manipulated by the learner in order to accomplish a more or less complex problem solution task. For example, a task might be to focus a cone of light using available objects such as an electric torch and a set of convex and concave lenses. An efficient, probabilistic assessment of skills in this context requires interpreting the learner's actions and, thus, the sequence of positions (and alignments) of all objects. The positions of all objects of a LeS constitute a problem solution state that can be evaluated with a specific utility function, determining the correctness of the current problem solution state, or in other terms, the learner's approach to the task's solution. In order to assess the available and lacking skills of the learner, the skills, which are necessary to correctly manipulate an object, must be assigned to each object. The probabilistic skill assessment occurs by updating an initial probability distribution over all skill states based on the utility value of the evaluation of the problem solution state. For example, if the learner uses the correct lens in order to focus a cone of light (i.e., a convex lens), the probabilities for skill states including the skill of knowing that convex lenses focus light, are increased. This procedure enables detecting the available and lacking skills of a learner. Moreover, on this basis also misconceptions (e.g., using a concave lens to focus light) or incorrect loops in the problem solution process (e.g., if the learners tries the same incorrect manipulations over and over again) can be detected. On this basis the system can determine suitable interventions (e.g., hints) for a specific problem solution state or sequence, which are directly related to the underlying latent skills.

\section{Conclusion}

The ELEKTRA project has the visionary goal of making a significant step towards educational games that can compete with commercial games in terms of immersion, engagement, and motivation while being a full educational option, utilizing appropriate balance between learning and gaming and relying on national curricula and sound psychological, pedagogical, and instructional theories. Developing a successful educational game, especially for older children, cannot be accomplished by adding some extra learning to a recreational game and it cannot be accomplished by adding some gaming to a traditional learning object. This aim requires the generation of a new genre within which both worlds, recreational games and psycho-pedagogical aims and methods, are meld. The adaptive technology which already exists and which is extended will help to fully incorporate the advantages of digital games for educational purposes and to make a significant step towards developing the interfaces for next-generation e-learning.

Acknowledgments. The research and development introduced in this work is funded by the European Commission under the sixth framework programme in the IST research priority, contract number 027986 . 


\section{References}

1. Merrill, M.D.: First principles of instruction. Educational Technology Research and Development 50 (2002), 43-59

2. Mitchell, A., Savill-Smith, C.: The use of computer and video games for learning: A review of the literature. Learning and Skills Development Agency, London (2004)

3. Malone, T.W.: Toward a theory of intrinsically motivating instruction. Cognitive Science 4 (1981) 333-369

4. Prensky, M.: Digital game-based learning. McGraw-Hill, New York (2001)

5. Schulmeister, R.: Didaktisches Design aus hochschuldidaktischer Sicht - Ein Plädoyer für offene Lernsituationen. In Rinn, U., Meister, D.M. (eds.): Didaktik und Neue Medien. Konzepte und Anwendungen in der Hochschule. Medien in der Wissenschaft 21 (2004) 19 $-49$

6. Van Eck, R.: Digital game-based learning: It's not just the digital natives who Are restless. Educause Review 41 (2006) 16-30

7. Koper, R., Olivier, B.: Representing the learning design of units of learning. Educational Technology \& Society 7 (2004) 97-111

8. Squire, K.: Cultural framing of computer/video games. Game Studies. The International Journal of Computer Game Research 1 (2002)

9. Gee, J.P.: What video games have to teach us about learning and literacy. Palgrave Macmillan, New York (2003)

10. Bloom, S.: Taxonomy of educational objectives, the classification of educational goals Handbook I: Cognitive Domain. McKay, New York (1956)

11. Leclercq, D., Poumay, M.: The 8 learning events Model. Release 2005. [Retrieved online February 5, 2007 from : http://www.labset.net/media/prod/8LEM.pdf]

12. Verpoorten, D.: The 8 learning events model: a pedagogic conceptual tool supporting diversification of learning methods. In: Proceedings of the 6th IEEE International Conference on Advanced Learning Technologies - (ICALT 2006) - Kerkrade, The Netherlands (2006)

13. Clark, J.M., Paivio, A.: Dual coding theory and education. Educational Psychology Review 3 (1991) 149-170

14. Crawford, C.: The art of computer game design. McGraw-Hill, Berkley (1984)

15. Ip, A., Morrison, I.: Learning objects in different pedagogical paradigms. In: Kennedy, G., Keppell, M., McNaught, C., Petrovic, T. (eds): Meeting at the crossroads: Proceedings 18th ASCILITE Conference. The University of Melbourne, Melbourne (2001) 289-298

16. Tyler, R.W.: Basic principles of curriculum and instruction. University of Chicago Press, Chicago (1950)

17. Mateas, M., Stern, A.: Façade, an artificial intelligence-based art/research experiment in electronic narrative [Retrieved February 5, 2007 from http://www.interactivestory.net/]

18. DeBra, P., Calvi, C.: AHA! An open Adaptive Hypermedia Architecture. The New Review of Hypermedia and Multimedia 4 (1998) 115-139

19. Csikszentmihalyi, M.: Flow: The psychology of optimal experience. Harper and Row, New York (1990)

20. Shernoff, D.J, et al.: Student engagement in high school classrooms from the perspective of flow theory. School Psychology Quarterly 18 (2003) 158-176

21. Doignon, J.-P., Falmagne, J.-C.: Knowledge spaces. Springer-Verlag, Berlin Heidelberg New York (1999)

22. Albert, D., Held, T.: Component based knowledge spaces in problem solving and inductive reasoning. In: Albert, D., Luks, J. (eds.): Knowledge Spaces: Theories, Empirical Research Applications. Lawrence Erlbaum Mahwah, NJ (1999) 15-40 
23. Doignon, J.-P.: Knowledge spaces and skill assignments. In Fischer, G., Laming, D. (eds.): Contributions to Mathematical Psychology, Psychometrics and Methodology. SpringerVerlag, Berlin Heidelberg New York (1994) 111-121

24. Düntsch, I., Gediga, G.: Skills and knowledge structures. British Journal of Mathematical and Statistical Psychology 48 (1995) 9-27

25. Korossy, K.: Extending the theory of knowledge spaces: a competence-performance approach. Zeitschrift für Psychologie 205 (1997) 53-82

26. Korossy, K.: Modelling knowledge as competence and performance. In: Albert, D., Luks, J. (eds.): Knowledge Spaces: Theories, Empirical Research Applications. Lawrence Erlbaum Mahwah, NJ (1999) 103-132 